\title{
DISEÑO DE UN GEMELO DIGITAL PARA EL GESTOR DE OPERACIONES DE UNA LAVANDERÍA INDUSTRIAL
}

\author{
Germán González Rodríguez \\ Dep. de Ingeniería Informática y de Sistemas, Universidad. de La Laguna, gcgonzal@ull.edu.es \\ José Manuel González Cava \\ Dep. de Ingeniería Informática y de Sistemas, Universidad de La Laguna, jgonzalc@ull.edu.es \\ Esteban Jove \\ Dep. Ingeniería Industrial, Universidade da Coruña, esteban.jove@udc.es \\ José Luis Calvo Rolle \\ Dep. Ingeniería Industrial, Universidade da Coruña, jlcalvo@udc.es \\ Juan Albino Méndez Pérez \\ Dep. de Ingeniería Informática y de Sistemas, Universidad de La Laguna, jamendez@ull.edu.es
}

\begin{abstract}
Resumen
En este trabajo se presenta una metodología para desarrollar un gemelo digital para un proceso industrial. El proceso es una lavandería industrial hospitalaria que puede ser caracterizada como una cadena de suministro de lazo cerrado. Una característica del proceso estudiado es la presencia de incertidumbre en la entrada, lo que dificulta la toma de decisiones en el proceso de producción. Para facilitar esta toma de decisiones se plantea el desarrollo de un gemelo digital basado en un sistema de lógica borrosa multinivel. La evaluación de la herramienta propuesta se ha hecho con datos reales obtenidos de una lavandería. Se ha comprobado que la propuesta ofrece resultados satisfactorios demostrando el potencial de esta herramienta en los procesos de producción.
\end{abstract}

Palabras clave: Gemelo digital, lógica difusa, cadenas de suministro en lazo cerrado, procesos de producción.

\section{INTRODUCCION}

La Fabricación Inteligente, considerada como la cuarta revolución industrial e identificada con el término Industria 4.0, es la colección de tecnologías de última generación que soportan la efectividad y la ingeniería de precisión en la toma de decisiones a tiempo real [5]. Industria 4.0 define una metodología para generar una transformación desde la fabricación dominada por máquinas a la fabricación digital [11]. Pero implantar nuevas tecnologías en los procesos industriales no es tarea sencilla, pues a la disponibilidad económica necesaria para la inversión, se suman factores logísticos y sociales que dificultan una reconversión inmediata. Por ello, elegir los caminos que desarrollen de manera paulatina la hoja de ruta de Industria 4.0, apoyándose inicialmente en los cambios en la gestión de operaciones, con menos requerimientos de implantación, parece una alternativa razonable para la mediana y pequeña industria.

Para cualquier Centro de Producción, el gestor del proceso buscará una solución adecuada a cada instante, de acuerdo a sus conocimientos y experiencia, y podrá: actuar sobre el ritmo de segregación de productos usados, externalizar algunas tareas para evitar colapsos, modificar la inyección de componentes nuevos o utilizar componentes ya procesados almacenados para la regulación de la cadena o para gestionar contingencias que pudiera tener la misma.

Pero estas variables, debido a las incertidumbres específicas de estos procesos señaladas en párrafos anteriores y a las propias de todo centro industrial también nombradas con anterioridad, generan situaciones muy diversas, que no sólo obligan a tener una formación específica importante en este entorno, sino además a tener ciertas facultades de clarividencia para detectar el estado de elementos intangibles, como los ritmos admisibles en cada momento que pueden alcanzar los recursos, especialmente los humanos, que no son siempre iguales por circunstancias infinitas.

Un prometedor camino para las compañías de manufacturación que buscan soportes para la toma de decisiones está en la digitalización de los elementos de producción, que en los últimos años se conoce como los Gemelos Digitales (Digital Twin) [6]. Estos modelos persiguen una simulación de alta escala, que 
use los mejores modelos físicos disponibles, datos de sensores y de históricos para construir un espejo de los sistemas reales [12]. Para muchos autores Digital Twin es el siguiente nivel de simulación y se convertirá en la principal herramienta en la ayuda a la toma de decisiones en el ámbito industrial, pues integrará datos de ingeniería, de operación y de comportamiento conectándolos a través de una arquitectura propia [1].

En este artículo se propone construir un espejo del gestor de producción de un centro de recuperación de productos usados, de manera que pueda replicar las decisiones sobre la programación de tareas que adopta este último en condiciones óptimas, sin estar sometido a las perturbaciones del entorno. Como caso de aplicación se considerará una lavandería industrial que representa un ejemplo claro de una cadena de suministro de lazo cerrado. El sistema resultante de ayuda a la toma de decisiones evitará el riesgo de una planificación de tareas no acertada como resultado de la complejidad propia del proceso y de la presencia de perturbaciones.

El documento comienza haciendo una revisión de aspectos relacionados con las cadenas de suministro de lazo cerrado. Posteriormente, se describe con detalle el proceso y se propone una solución para el problema planteado. Se finaliza con los resultados de la simulación propuesta y una discusión sobre los mismos.

\section{CADENAS DE SUMINISTRO EN LAZO CERRADO: CONCEPTOS BÁSICOS}

Una de las definiciones más reconocidas en la literatura académica [10] de una Cadena de Suministro (CS) es la que la propone como una "red de organizaciones que están implicadas a través de vínculos ascendentes y descendentes en los procesos y actividades que producen valor en forma de productos y servicios entregados al consumidor final" [2]. Si a esta definición tan extendida con la condición necesaria de recuperar valor de ese consumidor final, estaremos definiendo una Cadena de Suministro de Circuito Cerrado (CSCC). Esas dependencias entre organizaciones generan incertidumbres que es necesario manejar con acierto para acercarse al objetivo de eficiencia que se persigue.

Las cadenas de suministro de circuito cerrado se diferencian respecto a las tradicionales fundamentalmente en la necesidad de las primeras de recuperar un valor del cliente o usuario final [4]. En la gestión de operaciones en planta de procesos industriales integrados en cadenas de suministro, se debe contar con compromisos profundos tanto para la disposición de materias primas para la producción, como de finalización de productos acabados [14]. Pero estos compromisos en las cadenas de suministro de circuito cerrado se enfrentan a una gran variabilidad de situaciones posibles en el manejo de las existencias, es decir a una dinámica compleja, que va más allá incluso de las que tiene el propio proceso productivo. Esta conducción tan compleja de los sistemas productivos genera incertidumbres. Los ejemplos más comunes de incertidumbres están relacionados con el desconocimiento de la cantidad y proporción de los componentes que forman las materias primas a procesar. Por ello, a los actores no les resulta fácil encontrar la decisión que les lleve a la mejor estrategia para todos los casos. Estudios recientes han argumentado que las operaciones de las cadenas de suministro de circuito cerrado están limitadas por los efectos de esa dinámica compleja y por las incertidumbres [7].

En cada periodo productivo se toman decisiones para alinear los resultados deseados con el estado productivo observado (suministro de materias primas, eficiencia de los procesos programados, averías, incidencias relacionadas con personal, etc.). En las actuales empresas de fabricación de tamaños mediano y pequeño, estas decisiones las toman gestores de producción expertos de acuerdo con las estrategias empresariales, y a su experiencia y capacidad. Pero las múltiples variables a controlar, y los componentes no lineales de las mismas, impiden que dichas decisiones sigan siempre los criterios de las compañías, provocando desviaciones de cierta importancia de los resultados obtenidos respecto a los esperados, que mantenidas en el tiempo evidencian las deficiencias en el manejo del proceso. La no linealidad de las variables en una cadena de suministro se refiere al hecho de que los cambios en la entrada de una cadena pueden no estar relacionados proporcionalmente con los cambios en su producción [13].

\section{DESCRIPCIÓN DEL PROCESO}

Como caso de estudio se considerará la ayuda a la toma de decisiones en la gestión de operaciones para el control de las dotaciones, en el proceso de una lavandería industrial integrada en una Cadena de Suministro de Circuito Cerrado (CSCC), que tiene por objeto la reutilización de la ropa sucia hospitalaria.

El circuito de reutilización de la ropa hospitalaria, que constituye una CSCC, dispone de una red de organizaciones vinculadas por actividades que se desarrollan en ambos sentidos, que producen valor con el objetivo de ajustarse a la demanda de un consumidor final, del que se obtiene el producto una vez usado para su reutilización. Las organizaciones que intervienen son (ver Figura 1): la Unidad 
Asistencial Hospitalaria (UAH), el Centro Logístico Hospitalario (CLH), Lavandería Hospitalaria Industrial (LHI) y el Proveedor de Ropa Nueva (PRN).

\subsection{FASES DEL PROCESO DE PRODUCCIÓN}

Las tareas críticas en el proceso de producción son ([3]):

- Clasificación: segregación y clasificación de la ropa sucia por tipos de prendas cuyas secuencias, procedimientos, número de ciclos y tiempos de ejecución en la fase de procesado posterior a la clasificación sean idénticos.

- Procesado: lavado, secado, planchado y plegado.

- Expedición: empaquetado y definición de destinos. Los destinos pueden ser externos a la LHI, es decir a los CLH del Hospital al que corresponda la ropa reutilizada, o internos, a los Almacenes de Regulación o de Contingencias. Estos almacenes sirven para compensar los desequilibrios de ropa hospitalaria entre la demanda y la capacidad de entrega. El Almacén Regulador se emplea para completarlas dotaciones producidas en circunstancias normales de funcionamiento de la LHI. El segundo, el Almacén de Contingencias, es utilizado cuando se producen hechos excepcionales, como son las indisponibilidades imprevistas.

\subsection{TOMA DE DECISIONES DEL GESTOR}

Las variables con las que va a operar el gestor se muestran en la Tabla 1.

Tabla 1: Variables de operación del gestor.

\begin{tabular}{|l|l|}
\hline Variable & Designación \\
\hline Dotación procesada & DTpr \\
Dotación demandada & DTdm \\
Dotación de regulación & DTrg \\
Dotación de contingencia & DTcg \\
\hline
\end{tabular}

Los objetivos de producción de una LHI fundamentalmente son:

- Que la DTpr alcance como mínimo a la DTdm.

- Que el primer objetivo se realice con los recursos que la estrategia preestablecida de la compañía considera necesarios para lograr la DTpr.

- Que los niveles de las DTrg y DTcg se mantengan por encima de los mínimos establecidos.

- Que las condiciones de inicio de la producción del periodo siguiente al programado sean adecuadas, es decir, que no se encuentren situaciones de colapso.

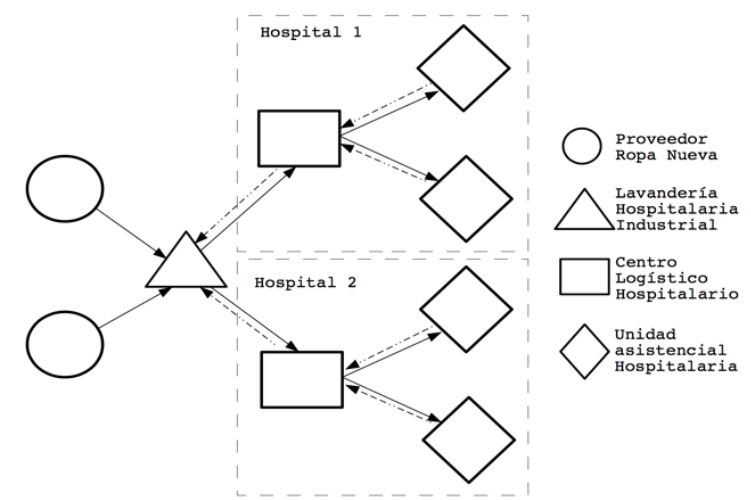

Figura 1. Representación de la cadena de suministro de lazo cerrado asociada a una lavandería hospitalaria industrial.

Para cumplir con estos cuatro objetivos el gestor de producción adoptará decisiones en instantes determinados del periodo programado, basadas en los datos de las variables que recibe correspondientes a el intervalo de tiempo que ha transcurrido desde la anterior toma de decisiones hasta ese momento. Los datos que dispondrá son de las variables siguientes: tiempo de producción disponible, producción programada pendiente, diferencias entre las cantidades de cada tipo de prenda que han entrado y salido del almacenamiento de ropa sucia clasificada y ritmo de segregación. Esta información la procesará y tras ello determinará un plan a muy corto plazo, a través del cual tratará de equilibrar el tiempo de producción disponible con la producción programada, utilizando de la manera más eficiente posible los recursos con los que cuenta. Para adoptar la mejor solución que siga la estrategia empresarial y evite los colapsos el gestor de producción, utilizando sus conocimientos y experiencia podrá: actuar sobre el ritmo de segregación de ropa sucia mezclada, externalizar algunas tareas para evitar colapsos, inyectar ropa nueva o utilizar la que está reservada lavada en los almacenes de Regulación o Contingencia.

Pero estas variables, debido a las incertidumbres específicas de este proceso señaladas en párrafos anteriores y a las propias de todo centro industrial también nombradas con anterioridad, generan situaciones muy diversas, que no sólo obligan a tener una formación específica importante en este entorno, sino además a disponer de ciertas facultades de clarividencia para detectar el estado de elementos intangibles, como los ritmos admisibles que pueden tener los recursos, especialmente los humanos, que no son siempre iguales por circunstancias diversas.

Por todo ello se persigue en este estudio, el diseño de elementos de ayuda a la toma de decisiones del gestor 
de la producción del proceso, que libere a éste en cierta medida de la presión de acertar en todos los momentos para cumplir tanto con el objetivo básico de la cadena de suministro, la efectividad, como con los criterios de eficiencia del centro de producción.

\section{DISEÑO DEL MODELO DIGITAL}

Este trabajo tiene como antecedente la optimización de un problema de Flow Shop Híbrido de uno de los procesos de la LHI, que se resolvió mediante programación matemática. Ese estudio previo sirvió para identificar un problema de un nivel superior, que tiene como origen la integración de ese centro de producción en una CSCC, que genera como se ha indicado con anterioridad, incertidumbres importantes en la gestión de las existencias, debido a la diversidad de situaciones que se pueden producir. Por ello, la presente propuesta tiene la intención de proporcionar un sistema de ayuda a las decisiones, que unifique las mismas de acuerdo a la política empresarial, eliminando las distorsiones que se introducen en las decisiones cuando éstas se adoptan por el gestor real en condiciones no ideales.

Para el diseño del gemelo digital se parte de la hipótesis de que en el proceso de producción existe un producto principal (generalmente la de mayor volumen de producción) para el que se deben definir las condiciones óptimas de producción y que el resto de productos se deben acomodar al ritmo de producción definido para este producto principal.

De esta manera el replicador inteligente del gestor de operaciones constará de dos niveles de decisión, un nivel principal o maestro y un nivel secundario o esclavo (ver figura 2). Dada las características de los sistemas basados en lógica borrosa es común su aplicación para el modelado y control de sistemas complejos en diversas áreas de aplicación ([8], [9]). La implementación de estos sistemas de decisión se hará mediante lógica borrosa, de tal modo que se tendrá un sistema de soporte a decisiones (DSS) basado en lógica borrosa para cada uno de los productos.

En el caso estudiado el producto principal será el procesado de sábanas. El periodo programado para dar soporte a las decisiones es de ocho horas, que coincide con un turno de producción de la planta real estudiada. El decisor principal, DSSs, adopta la decisión más importante para el gestor: establecer el ritmo de procesamiento de la planta en el periodo productivo programado. Además de esta decisión tomará decisiones asociadas al producto específico que gobierna. El sistema incluye en el nivel secundario, un $D S S$ por cada una de las prendas restantes que procese la LHI (mantas, colchas, toallas y pijamas). Estos toman las decisiones operativas sobre cada una de ellas, pero están condicionados por el ritmo de producción marcado por el DSSs. Cada uno de los DSS consta de un Sistema de Inferencia Borrosa (FIS) para cada una de las prendas que se están procesando. Las decisiones operativas sobre las prendas de los FIS indicados anteriormente serán: externalizar una parte de la producción, utilizar el almacén regulador para completar la producción, inyectar ropa nueva, o producir con exceso para dotar los almacenes de la LHI. El gestor real adopta estas decisiones en función de la información que recibe de la evolución de la producción. Su réplica digital también analizará la situación productiva del momento de toma de decisiones y dará respuestas simultáneas a través de los diferentes FIS. La información de entrada a estos DSS es la siguiente:

- $\quad R k$ : ritmo de producción actual.

- tr: tiempo de producción disponible del periodo a programar.

- Pri: producción pendiente de la prenda i (donde $i$ puede tomar los valores s (sábanas), $m$ (mantas), $c$ (colchas), $t$ (toallas) o $p$ (pijama).

- $\quad e i$ : diferencia entre entradas y salidas del almacén de clasificación de ropa sucia de la prenda $i$.

Las salidas de los sistemas de inferencia borrosa son:

- $\quad$ Rkl: ritmo de producción a aplicar.

- $\quad I i$ : inyección de ropa de tipo $i$.

- $R i$ : aporte desde almacén regulador de ropa de tipo $i$.

- $E i$ : externalización para provisión de ropa de tipo $i$.

Se ha empleado inferencia de tipo Takagi-Sugeno para los sistemas de inferencia difusos planteados. Las funciones de pertenencia de las entradas son trapezoidales para los extremos del rango y triangulares para el resto. El operador AND empleado en el conjunto de reglas se ha establecido como la operación producto entre los valores borrosos de entrada. El método de defuzzificación elegido es la media ponderada de todas salidas activas. El resto de características específicas para cada FIS aparece especificado en la Tabla 2. Un ejemplo de la curva de superficie generada para la decisión de inyección puede verse en la Figura 4.

Tabla 2. Descripción de las distintas configuraciones de los sistemas de inferencia difusos planteados.

\begin{tabular}{|c|c|c|c|}
\hline \multirow{2}{*}{ FIS } & Entradas & $\begin{array}{c}\mathbf{N}^{\mathbf{0}} \\
\text { funciones } \\
\text { pertenencia }\end{array}$ & $\begin{array}{c}\mathbf{N}^{\mathbf{0}} \\
\text { funciones } \\
\text { salida }\end{array}$ \\
\hline \multirow{3}{*}{ Externalizar } & $t r$ & 7 & \multirow{2}{*}{28} \\
\cline { 2 - 3 } & $e$ & 10 & \multirow{2}{*}{28} \\
\cline { 2 - 3 } & $P r$ & 10 & \multirow{2}{*}{23} \\
\cline { 2 - 3 } Inyección & $R k 1$ & 11 & \multicolumn{2}{|c}{23} \\
\cline { 2 - 3 } & $e$ & 14 & \multicolumn{2}{|c}{} \\
\hline
\end{tabular}




\begin{tabular}{|l|c|c|c|}
\hline \multirow{4}{*}{ Regulador } & $P r$ & 9 & \multirow{2}{*}{} \\
\cline { 2 - 3 } & $R k 1$ & 7 & \multirow{2}{*}{2} \\
\cline { 2 - 3 } & $t r$ & 9 & \multirow{2}{*}{22} \\
\cline { 2 - 3 } & $e$ & 6 & 8 \\
\cline { 2 - 3 } $\begin{array}{l}\text { Ritmo } \\
\text { Clasificación }\end{array}$ & $\operatorname{tr} 1$ & 9 & \multirow{2}{*}{42} \\
\hline
\end{tabular}

De este modo el gemelo digital, que reproduce las decisiones del gestor de producción, vendrá definido por las salidas de este sistema borroso.

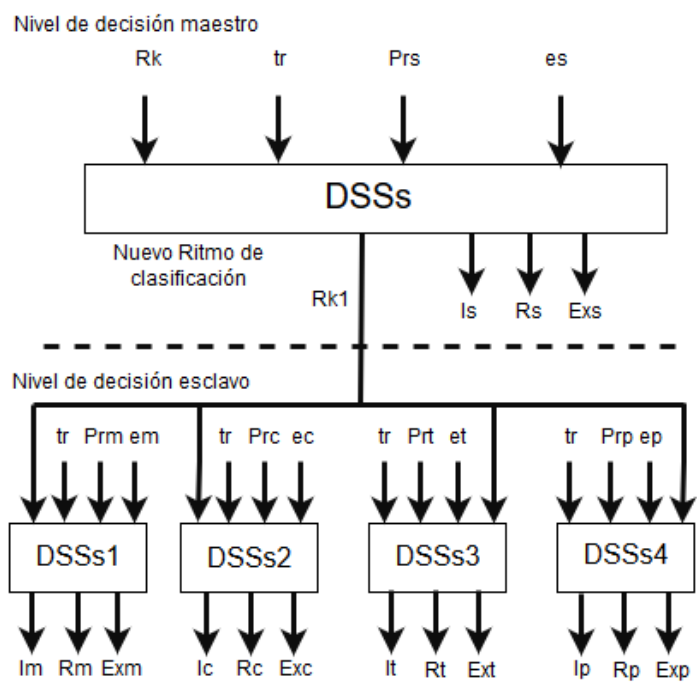

Figura 2. Sistema de ayuda a la toma de decisiones multinivel basado en inferencia borrosa. Variables: Rk (Ritmo de clasificación actual), Rk1 (nuevo ritmo de clasificación), tr (tiempo restante), e (diferencia entre demanda y entrada), Ii (inyección de ropa de tipo i), Ri (aporte desde almacén regulador de ropa de tipo i), Exi (externalización para provisión de ropa de tipo i). Los tipos de ropa son s (sábanas), $m$ (mantas), $c$ (colchas), $p$ (pijamas).

\subsection{EVALUACIÓN DEL MODELO}

Para evaluar el rendimiento del sistema propuesto se ha considerado un conjunto de datos adicional en el que se recogen los pares formados por el estado de producción y las decisiones asociadas propuestas por un experto real.

Se ha considerado entonces el error de decisión como la diferencia entre la solución aportada por el gemelo digital y la solución propuesta por el experto.

Siguiendo este procedimiento se analizan las variables Externalización, Inyección y Almacén Regulador, estimando para cada una de ellas los porcentajes de error y su incidencia.
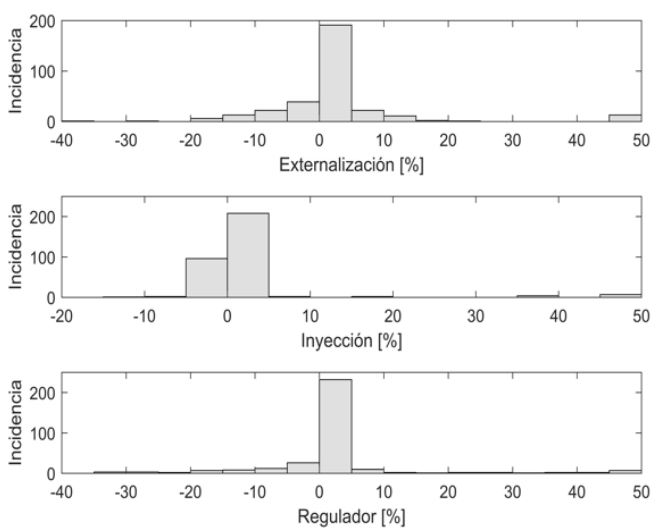

Figura 3. Gráfica de errores de la predicción de las variables referidas a la externalización, inyección y regulador para los sistemas de apoyo a la decisión propuestos

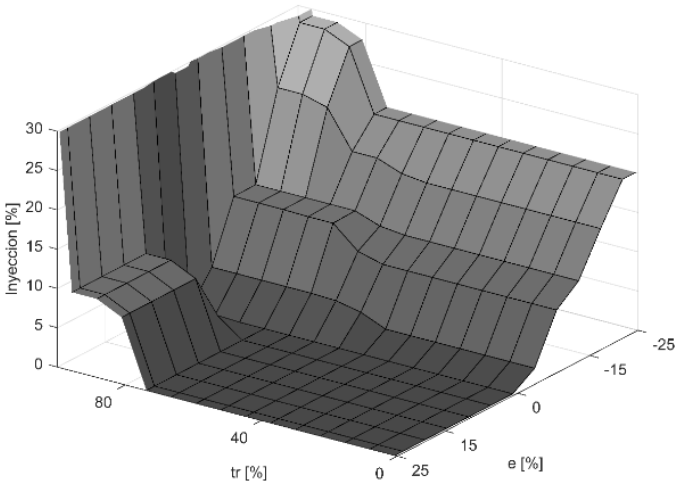

Figura 4. Curva de superficie generada a partir del sistema de inferencia difuso para la decisión de Inyección, considerando como entradas las variables tr ye.

\section{RESULTADOS}

En el caso de estudio se ha tratado de replicar al gestor de operaciones en planta de una Lavandería Hospitalaria Industrial (LHI), construyendo su Gemelo Digital. El gestor real dispondrá de esta herramienta de ayuda a la toma de decisiones, que propondrá soluciones alineadas con la estrategia empresarial para lograr la eficiencia productiva esperada.

El sistema se ha implementado usando la estructura mostrada en la Figura 2. El ajuste de los sistemas de inferencia borrosa se ha realizado a partir de un conjunto de datos que incluye el estado de la producción a lo largo de diferentes instantes de tiempo durante la jornada junto con las decisiones adoptadas en cada situación. Las pruebas del algoritmo resultante 
se han ejecutado en un ordenador PC basado en procesador i7 con 16 GB de RAM. Se ha usado el entorno Matlab ${ }^{\mathrm{TM}}$ para el preprocesado de los datos $\mathrm{y}$ la implementación del sistema de inferencia borrosa multinivel. Los tiempos de ejecución han sido del orden de milisegundos.

En la Figura 3 se presentan los resultados obtenidos para este error para las diferentes decisiones. Como puede observarse el comportamiento obtenido por el gemelo digital se aproxima de forma muy adecuada a las decisiones reales tomadas por el experto.

Se ha considerado entonces el error de decisión como la diferencia entre la solución aportada por el gemelo digital y la solución propuesta por el experto. En la Figura 3 se presentan los resultados obtenidos para este error para las diferentes decisiones. En general, se observa que para la mayoría de las decisiones el error cometido por el sistema de inferencia difuso es muy próximo a cero. Este comportamiento evidencia que la propuesta de gemelo digital desarrollada en este estudio se ajusta de manera satisfactoria al comportamiento real que cabría esperar de un experto en la toma de decisiones. Por otro lado, se observan algunas situaciones minoritarias en las que el error aumenta de forma atípica, alcanzando incluso valores del $50 \%$. Tras analizar estos casos de manera pormenorizada, se ha llegado a la conclusión de que se trata de situaciones puntuales incluidas en la validación que divergen del comportamiento esperado del sistema en condiciones de producción normal. Cabe destacar que las técnicas de Inteligencia Artificial empleadas en este estudio se basan en la minimización del error de validación cruzada de entrenamiento, generando para ello reglas difusas capaces de aglutinar el comportamiento general del sistema. Como consecuencia, los sistemas de inferencia difusos propuestos presentan una mayor dificultad para aprender situaciones atípicas que representan un número minoritario de muestras en el entrenamiento. Para corregir estas divergencias, y teniendo en cuenta la incidencia y posibles riesgos de cada una de las situaciones atípicas registradas, podrían definirse de manera manual y con la ayuda de un experto una serie de reglas para ser añadidas al conjunto de reglas original con vistas a minimizar los posibles efectos negativos sobre las decisiones.

Estos resultados soportarían la hipótesis de que la implementación de este algoritmo como una herramienta en tiempo real, podría ser de gran utilidad para la toma de decisiones en procesos de producción de lazo cerrado.

\section{CONCLUSIONES}

En este trabajo se ha propuesto el desarrollo de un gemelo digital para un gestor de operaciones de producción en una planta industrial. Se ha considerado el caso de una lavandería industrial, que es un ejemplo de cadena de suministro de lazo cerrado con perturbaciones en la entrada. La complejidad inherente de este tipo de sistemas justifica la necesidad de herramientas que faciliten la toma de decisiones en la planta.

Se ha analizado el proceso de producción y se ha planteado una estructura de decisión de dos niveles para el problema. A pesar de que el trabajo se centra en una lavandería industrial, esta estructura puede ser aplicada a otros procesos de lazo cerrado con perturbaciones.

Los resultados preliminares obtenidos permiten evidenciar el potencial y el interés de este tipo de metodologías para facilitar la toma de decisiones en este tipo de procesos industriales. 


\section{English summary}

\section{DESING OF A DIGITAL TWIN FOR THE OPERATIONS MANAGER OF AN INDUSTRIAL LAUNDRY.}

\begin{abstract}
This paper presents a methodology to develop a digital twin for an industrial process. The process is a hospital industrial laundry, that can be characterized as a Closed-loop supply chain. A characteristic of the process studied is the presence of uncertainty in the input, which makes decision making in the production process difficult. To facilitate this decision making, the development of a Digital Twin based on a multilevel fuzzy logic system is considered. The evaluation of the proposed tool has been done with real data obtained from a laundry. It has been proven that the proposal offers satisfactory results, demonstrating the potential of this tool in the production processes.
\end{abstract}

Keywords: Digital Twin, Fuzzy Logic, Closed-loop Supply Chain, Production processes.

\section{Agradecimientos}

Este trabajo ha sido financiado por Fundación CajaCanarias (proyecto Greentourist 2016TUR17).

\section{Referencias}

[1] Bricogne, M., Le Duigou, J., \& Eynard, B. (2016). Design Processes of Mechatronic Systems. In P. Hehenberger \& D. Bradley (Eds.), Mechatronic Futures: Challenges and Solutions for Mechatronic Systems and their Designers (pp. 75-89). Cham: Springer International Publishing. https://doi.org/10.1007/978-3-31932156-1 6.

[2] Christopher, Martin L. (1992), Logistics and Supply Chain Management, London: PitmanPublishing.

[3] González Rodriguez, G. C.; Méndez, J.A.; Belén Melián; González Cava, J.M.. A fuzzy modelling approach to laundry industry. Advances in Intelligent Systems and Computing. 642, Springer Nature. htts://doi.org/10.1007/978-3319-66824-6 14. 2017.

[4] Guide, V. D. R., Harrison, T. P., \& Van Wassenhove, L. N. (2003). The challenge of closed-loop supply chains. Interfaces, 33(6), 36.

[5] Kang, H. S., Lee, J. Y., Choi, S., Kim, H., Park, J. H., Son, J. Y., ... Noh, S. Do. (2016). Smart manufacturing: Past research, present findings, and future directions. International Journal of Precision Engineering and Manufacturing-Green Technology, $3(1)$ $111-128$ https://doi.org/10.1007/s40684-016-0015-5.
[6] Kunath, M., \& Winkler, H. (2018). Integrating the Digital Twin of the manufacturing system into a decision support system for improving the order management process. Procedia CIRP, 72, 225-231. https://doi.org/10.1016/j.procir.2018.03.192

[7] Linder, M., \& Williander, M. (2017). Circular Business Model Innovation: Inherent Uncertainties. Business Strategy and the Environment, 26(2), 182-196. https://doi.org/10.1002/bse.1906

[8] Méndez Pérez, J.A.; León, A.; Marrero, A.; González Cava, J. M.; Reboso, J.A.; Estévez Damas, J. I.; Gómez, J. F. Improving the anesthetic process by a fuzzy rule based medical decision system. Artificial Intelligence In Medicine. Elsevier, 2018. ISSN 0933-3657

[9] Méndez, J. A.; González, E. A control system proposal for engineering education. Computers \& Education. 68, pp. 266 - 274. Elsevier, 2013. ISSN 0360-1315

[10] Mentzer, J. T., DeWitt, W., Keebler, J. S., Min, S., Nix, N. W., Smith, C. D., \& Zacharia, Z. G. (2001). Defining Supply Chain Management. Journal of Business Logistics, 22(2), 1-25. https://doi.org/10.1002/j.21581592.2001.tb00001.x

[11] Oztemel, E., \& Gursev, S. (2018). Literature review of Industry 4.0 and related technologies. Journal of Intelligent Manufacturing, (January). https://doi.org/10.1007/s10845-018-1433-8.

[12] Shafto M., Conroy M., Doyle R., Glaessgen E. H., Kemp C., LeMoigne J, Wang L. DRAFT Modeling, Simulation, Information Technology \& Processing Roadmap - Technology Area 11. Washington, DC: National Aeronautics and Space Administration, 2010.

[13] Surana, A., S. K., Greaves, M., \& Raghavan, U. N. (2005). Supply-chain networks: a complex adaptive systems perspective. International Journal of Prod. Research, 43(20), 4235-4265. https://doi.org/10.1080/00207540500142274.

[14] Xu, W., Song, D., \& Roe, M. (2011). Production and raw material ordering management for a manufacturing supply chain with uncertainties. IEEE International Conference on Industrial Engineering and Engineering Management, 747-751. https://doi.org/10.1109/IEEM.2011.6118016.

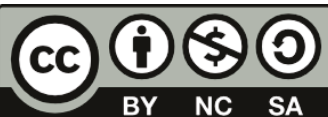

(C) 2019 by the authors. Submitted for possible open access publication under the terms and conditions of the Creative Commons Attribution CC BY-NC-SA 4.0 license (https://creativecommons.org/licenses/bync-sa/4.0/deed.es). 